\title{
Analysis of highly nonlinear oscillation systems using He's max-min method and comparison with homotopy analysis and energy balance methods
}

\author{
L B IBSEN $^{1}$, A BARARI $^{1, *}$ and A KIMIAEIFAR ${ }^{2}$ \\ ${ }^{1}$ Department of Civil Engineering, Aalborg University, Sohngårdsholmsvej 57, \\ 9000 Aalborg, Aalborg, Denmark \\ ${ }^{2}$ Department of Mechanical Engineering, Aalborg University, \\ Pontoppidanstraede 101, DK-9220 Aalborg East, Denmark \\ e-mail: ab@civil.aau.dk; amin78404@yahoo.com
}

MS received 29 January 2010; revised 27 April 2010; accepted 1 June 2010

\begin{abstract}
Nonlinear functions are crucial points and terms in engineering problems and the solutions of many important physical problems are centered on finding accurate solutions to these functions. In this paper, a new method called max-min method has been presented for deriving accurate/approximate analytical solution to strong nonlinear oscillators. Furthermore, it is shown that a large class of linear or nonlinear differential equations can be solved without the tangible restriction of sensitivity to the degree of the nonlinear term, adding that the method is quite convenient due to reduction in size of calculations. Results obtained by max-min are compared with Homotopy Analysis Method (HAM), energy balance and numerical solution and it is shown that, simply one term is enough to obtain a highly accurate result in contrast to HAM with just one term in series solution. Finally, the phase plane to show the stability of systems is plotted and discussed.
\end{abstract}

Keywords. Min-max method; nonlinear oscillation; duffing equation; homotopy analysis method; He's energy balance method.

\section{Introduction}

Most of engineering problems, especially some oscillation equations are nonlinear, and in most cases it is difficult to solve such equations, especially analytically. Perturbation method is one of the well-known methods to solve nonlinear problems; it is based on the existence of small/large parameters, the so-called perturbation quantity (Bender et al 1989; He 2002a).

Recently, considerable attention has been paid towards approximate solutions for analytically solving nonlinear differential equation. Many nonlinear problems do not contain such perturbation quantity, so to overcome the shortcomings, many new techniques have appeared

*For correspondence 
in open literature such as; harmonic balance (Hamdan \& Shabaneh 1997), homotopy perturbation (Barari et al 2008; He 2004; Özis \& Yildirim 2007a), modified Lindstedt-Poincaré (He 2001; He 2002b; Özis \& Yildirim 2007b; Mohammadi et al 2009), Adomian decomposition (Dehghan \& Shakeri 2008), amplitude-frequency formulation (Ganji et al 2009), parameterexpansion (Kimiaeifar et al 2010), parameterized perturbation (He 1999a), multiple scale (Okuizumi \& Kimura 2004), energy balance (He 2006a; Momeni et al 2010), variational approach (He 2007; Xu 2008; Tolou et al 2009; Hashemi Kachapi et al 2009), Variational Iteration (He et al 2010; Herisanu \& Marinca 2010), Newton-harmonic balancing (Lai et al 2009), differential transformation (Catal 2008), max-min (He 2008; Zeng \& Lee 2009; Zeng 2009; Shen \& Mo 2009).

In this paper, the basic idea of max-min method is introduced and then its applications in some nonlinear oscillators are studied. In most of the engineering problems, it is easy to find maximum/minimum thresholds of a solution of a nonlinear equation. In max-min method the angular frequency can be readily obtained. The max-min method (He 2008) was proposed by Ji-Huan He, based on ancient Chinese mathematics (Qian 1992; Ji 1999). The results are valid not only for weakly nonlinear systems, but also for strongly nonlinear ones. To show the accuracy of solutions, results are compared with those obtained by energy balance, homotopy analysis and numerical methods.

\section{Max-min approach (MMA)}

The max-min method arose from an ancient Chinese inequality, called He Chengtian's Inequality (He 2009) and proposed by $\mathrm{He}$ (2008). Chengtian inequality was mainly used to find the fractional day of the Moon by using the strong factor of 15 and the weak factor of 1 in the following form:

$$
29 \frac{26}{49} \text { days }>1 \text { moon }>29 \frac{9}{17} \text { days. }
$$

Using the weighting factors (15 and 1), He Chengtian obtains the following fractional day:

$$
\text { The fractional day }=\frac{26 \times 15+9 \times 1}{49 \times 15+17 \times 1}=\frac{399}{752} .
$$

So

$$
1 \text { moon }=29 \frac{399}{752} \text { days. }
$$

He Chengtian actually uses the following inequality:

If

$$
\frac{a}{b}<x<\frac{d}{c},
$$

where $a, b, c$ and $d$ are real numbers, then

$$
\frac{a}{b}<\frac{m a+n d}{m b+n c}<\frac{d}{c},
$$

and $x$ is approximated by:

$$
x=\frac{m a+n d}{m b+n c},
$$

where $m$ and $n$ are weighting factors. 


\section{Basic idea of max-min method}

Based on the MMA the Duffing equation is rewritten, in the following form:

$$
\ddot{v}+\omega^{2} v=0
$$

where $\omega^{2}=N(v, \dot{v}, \ddot{v}, t) / v$.

Then, the maximum and minimum for $\omega^{2}$ is calculated. Note that, there is no commitment to use the maximum and the minimum amount of $\omega^{2}$ and the only necessary thing is an upper and a lower boundary, if they are available. It is axiomatic that finding boundaries with less variance will lead to a more accurate approximation. In the limit state, to find the maximum and the minimum of $\omega^{2}$, a trial function is used. Choosing an appropriate trial function is another factor which will help to a better approximation for MMA, especially in the case of non-conservative oscillators. A well known trial functions $A \cos \omega t$ or $A \sin \omega t$ for conservative oscillators is used, according to the initial condition. To find the maximum and minimum, the maximum and minimum amount of the trial functions in $\omega^{2}$ is substituted.

Based on the He Chengtian's Inequality, the frequency value will identify as:

$$
\frac{a}{b}<\omega^{2}=\frac{m a+n d}{m b+n c}<\frac{d}{c}
$$

where $\frac{a}{b}$ and $\frac{d}{c}$ are the lower and the upper boundary of $\omega^{2}$ or the maximum and the minimum amount. $a, b, c$ and $d$ are real numbers and $m, n$ are weighting factors. So, it is obtained:

$$
\ddot{v}+\omega^{2} v=\ddot{v}+N(v, \dot{v}, \ddot{v}, t)+\Omega(v, \dot{v}, \ddot{v}, t)
$$

and then, to find the solution a Fourier expansion series is set

$$
\Omega(v, \dot{v}, \ddot{v}, t)=0 .
$$

If, by chance, (8) is the exact angular frequency, then the right hand side of (9) is vanishing completely. It is interesting to note that $m$ and $n$ can also be functions of time in which finding a right weighting factor then (8) happens to be the exact solution. This is the third factor which will help someone in finding a more accurate solution when using MMA.

\section{Application to nonlinear oscillators}

In order to assess the advantages and the accuracy of the max-min method, the following three examples are considered:

\subsection{Example 1}

First, the Duffing equation is considered (He 2006b):

$$
u^{\prime \prime}+c^{2} u+\varepsilon u^{3}=0, \quad u(0)=A, \quad u^{\prime}(x)=0,
$$

where $c$ and $\varepsilon$ are the linear and cubic stiffness which do not need to be small in the present study, $0 \leq \varepsilon<\infty, c>1$.

By rewriting (11) in the following form:

$$
u^{\prime \prime}+\left(c^{2}+\varepsilon u^{2}\right) u=0 .
$$


According to the initial conditions, a trial-function in the form of the following equation is chosen, which can satisfy the boundary conditions.

$$
u=A \cos \omega t,
$$

where $\omega$ is the frequency to be determined. It is clear that the square of frequency and $\omega^{2}$ is never less than that in the solution, and never exceeds the square of frequency of the solution. From (13), therefore:

$$
-\omega^{2} u-c^{2}+\varepsilon u^{3}=0, \Rightarrow \omega^{2}=-c^{2}+\varepsilon u^{2}, \Rightarrow \omega=\sqrt{\left(\varepsilon A^{2} \cos ^{2} \omega t-c^{2}\right)} .
$$

From (14), it is obtained:

$$
\varepsilon A^{2} \cos ^{2} \omega t>c^{2} \Rightarrow \sqrt{\varepsilon}|A| \cos \omega t>|c| \Rightarrow \sqrt{\varepsilon}\left|\frac{A}{c}\right| \cos \omega t>0
$$

and

$$
0<\omega^{2}<\varepsilon A^{2}-c^{2}
$$

According to He Chengtian's interpolation, we have:

$$
\omega^{2}=\frac{m(0)+n\left(\varepsilon A^{2}-c^{2}\right)}{m+n}=k\left(\varepsilon A^{2}-c^{2}\right),
$$

where $m$ and $n$ are weighting factors, $k=n /(m+n)$; so the frequency can be approximated as:

$$
\omega=\sqrt{\left(k\left(\varepsilon A^{2}-c^{2}\right)\right)} .
$$

Its approximate solution reads:

$$
u(t)=A \cos \left[\left(k\left(\varepsilon A^{2}-c^{2}\right)\right)^{1 / 2} t\right],
$$

by rewriting (11), adding and subtracting the term $k \varepsilon A^{2} u$ it is obtained:

$$
u^{\prime \prime}-c^{2} u+k \varepsilon A^{2} u=k \varepsilon A^{2} u-\varepsilon u^{3} .
$$

If (19) is the exact solution, then the right hand side of (20) is vanishing completely. Since the approach is only an approximation to the exact solution, it is set:

$$
B=\int_{0}^{T / 4}\left[k \varepsilon A^{2} u-\varepsilon u^{3}\right] \cos \omega t d t=0
$$

by substituting (19) into (21), where $T=2 \pi / \omega$, it is obtained as:

$$
\left\{\begin{array}{l}
B_{1}=\int_{0}^{T / 4} k \varepsilon A^{3} \cos ^{2} \omega t d t=\int_{0}^{\pi / 2} k \varepsilon A^{3}\left(\frac{1+\cos 2 \omega t}{2}\right)=\left[k \varepsilon A^{3}\left(\frac{x}{2}+\frac{\sin 2 \omega t}{2}\right)\right]_{0}^{\pi / 2} \\
B_{2}=\int_{0}^{T / 4}-\varepsilon A^{3} \cos ^{4} \omega t d t=\int_{0}^{\pi / 2} \frac{1}{4}+\frac{\cos 2 \omega t}{2}+\frac{1}{8}+\frac{\cos 4 \omega t}{8}
\end{array}\right.
$$


Because of two terms in the above integral equation, they are solved separately, and supposed $B=B_{1}+B_{2}$.

$$
\begin{aligned}
& \left\{\begin{array}{l}
B_{1}=k \varepsilon A^{3} \frac{\pi}{4} \\
B_{2}=\left(\frac{\pi}{4}+\frac{\pi}{16}\right) \varepsilon A^{3}
\end{array} \Rightarrow B=B_{1}+B_{2}=k \varepsilon A^{3} \frac{\pi}{4}-\frac{3 \pi}{16} \varepsilon A^{3}\right. \\
& B=\varepsilon A^{3} \frac{\pi}{4}\left(k-\frac{3}{4}\right)=0 .
\end{aligned}
$$

Therefore,

$$
k=3 / 4 \text {. }
$$

The obtained frequency is:

$$
\omega=\sqrt{\left(\frac{3}{4} \varepsilon A^{2}-\frac{3}{4} c^{2}\right)} .
$$

In addition, the approximate period is:

$$
T=\frac{2 \pi}{\sqrt{\left(\frac{3}{4} \varepsilon A^{2}-\frac{3}{4} c^{2}\right)}} .
$$

To show the remarkable accuracy of the obtained result, the approximate period with the He's Energy Balance Method (Babazadeh et al 2008) and HAM are compared in table 1 and figure 1 .

Finally, for selected constant parameters, the stability of the system is shown in figure 2. Generally, the system possesses no damping, and therefore, any excitation will cause instability of the system.

Table 1. Comparison between different methods to obtain the frequency $(\varepsilon=0 \cdot 2, c=0 \cdot 5)$.

\begin{tabular}{lccccc}
\hline A & $\begin{array}{c}\text { Energy balance } \\
\text { frequency }\end{array}$ & $\begin{array}{c}\text { Max-min } \\
\text { frequency }\end{array}$ & $\begin{array}{c}\text { HAM with } \\
1 \text { order }\end{array}$ & $\begin{array}{c}\text { HAM with } \\
10 \text { orders }\end{array}$ & $\begin{array}{c}\text { Numerical } \\
\text { solution }\end{array}$ \\
\hline 4 & 1.46629 & 1.48745 & 1.32702 & 1.45394 & 1.45375 \\
$4 \cdot 1$ & 1.50715 & 1.52774 & 1.38806 & 1.50015 & 1.50055 \\
4.2 & 1.54790 & 1.56796 & 1.41792 & 1.53192 & 1.53103 \\
$4 \cdot 3$ & 1.58855 & 1.60810 & 1.44781 & 1.57196 & 1.57076 \\
4.4 & 1.62911 & 1.64818 & 1.50901 & 1.62013 & 1.62027 \\
4.5 & 1.66958 & 1.68819 & 1.51459 & 1.65929 & 1.65992 \\
4.6 & 1.70997 & 1.72815 & 1.54951 & 1.68929 & 1.68901 \\
4.7 & 1.75029 & 1.76805 & 1.59910 & 1.74902 & 1.74967 \\
4.8 & 1.79053 & 1.80790 & 1.61283 & 1.77182 & 1.77165 \\
4.9 & 1.83071 & 1.84770 & 1.69192 & 1.81518 & 1.81508 \\
5 & 1.87083 & 1.88746 & 1.75192 & 1.84926 & 1.84915 \\
\hline
\end{tabular}




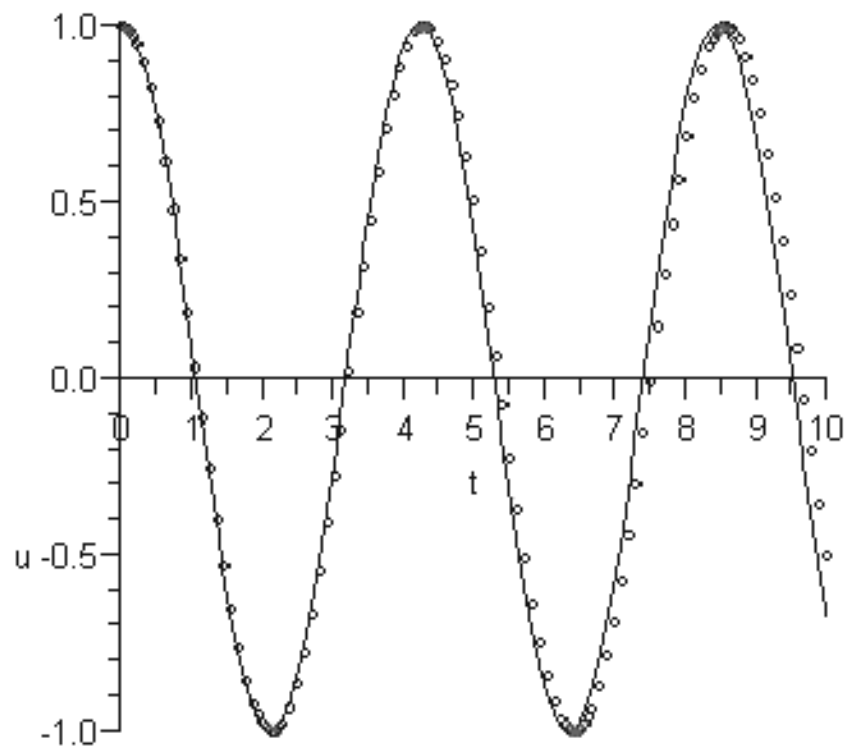

Figure 1. Comparison of the energy balance solution with the max-min solution; pointed line; energy balance and solid line; the max-min solution. Example 1: $(A=4, c=0 \cdot 5)$.

4.1a HAM Solution for Example 1: Nonlinear operator for the example 1 is defined as follows (Kimiaeifar et al 2009a):

$$
N[u(t ; q)]=\frac{\partial^{2} u(t ; q)}{\partial t^{2}}+c^{2} u(t ; q)+\varepsilon u(t ; q)^{3},
$$

where $q \in[0,1]$ is the embedding parameter. As the embedding parameter increases from 0 to $1, U(t ; q)$ varies from the initial guess, $U_{0}(t)$, to the exact solution, $U(t)$.

$$
u(t ; 0)=U_{0}(t), \quad u(t ; 1)=U(t) .
$$

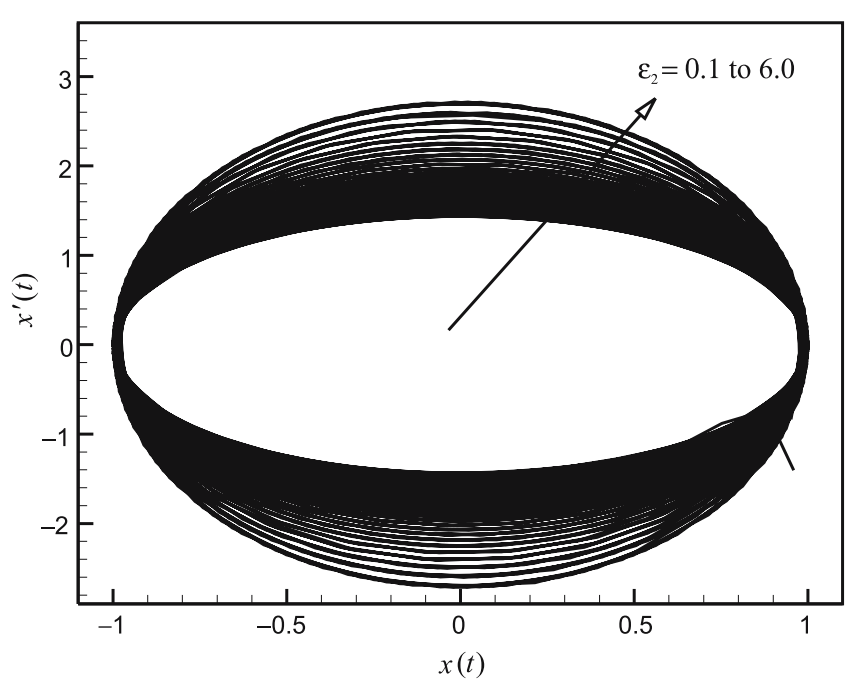

Figure 2. Phase plane maps showing the system stability, influence of $\varepsilon$ in the stability. 
Expanding $x(t ; q)$ in Taylor series with respect to $q$ results in:

$$
u(t ; q)=U_{0}(t)+\sum_{m=1}^{\infty} U_{m}(t) q^{m}
$$

where

$$
U_{m}(t)=\left.\frac{1}{m !} \frac{\partial^{m} u(t ; q)}{\partial q^{m}}\right|_{q=0} .
$$

Homotopy analysis method can be expressed by many different base functions (Kimiaeifar et al 2009b), according to the governing equations; it is straightforward to use a base function in the form of:

$$
U(t)=\sum_{m=1}^{\infty} \sum_{p=1}^{\infty} b_{k p m} t^{k} \cos ^{m}(t) \sin ^{p}(t),
$$

that $b_{k p m}$ are the coefficients to be determined. When the base function is selected, the auxiliary functions $H(t)$, initial approximations $U_{0}(t)$ and the auxiliary linear operators $L$ must be chosen in such a way that the corresponding high-order deformation equations have solutions with the functional form similar to the base functions. This method referred to as the rule of solution expression (Fooladi et al 2009; Kimiaeifar 2010).

The linear operator $L$ is chosen as:

$$
L[u(t ; q)]=\frac{\partial^{2} u(t ; q)}{\partial t^{2}}+u(t ; q) .
$$

From equation (31) it can be obtained as

$$
L\left[c_{1} \sin (t)+c_{2} \cos (t)\right]=0,
$$

where $c_{1}$ to $c_{2}$ are the integral constants. According to the rule of solution expression and the initial conditions, the initial approximations, $U_{0}(t)$ as well as the integral constants, $c_{1}$ to $c_{2}$ are formed as:

$$
U_{0}(t)=c_{1} \sin (t)+c_{2} \cos (t), \quad c_{1}=0, c_{2}=\lambda .
$$

The zeroth order deformation equation for $U(t)$ is:

$$
\begin{aligned}
& (1-q) L\left[u(t ; q)-U_{0}(t)\right]=q \hbar H(t) N[u(t ; q)], \\
& u(0 ; q)=A, \quad \frac{\partial u(0 ; q)}{\partial t}=0 .
\end{aligned}
$$

According to the rule of solution expression and from equation (34), the auxiliary function $H(t)$ can be chosen as follows (Fooladi et al 2009):

$$
H(t)=1
$$


Differentiating equation 16, $m$ times, with respect to the embedding parameter $q$ and then setting $q=0$ in the final expression and dividing it by $m$ !, it is reduced to:

$$
\begin{aligned}
U_{m}(t)= & \chi_{m} U_{m-1}(t)+\eta\left(\sin (t) \int_{0}^{t} H(t) R_{m}\left(U_{m-1}\right) \cos (t) d t\right. \\
& \left.+\cos (t) \int_{0}^{t} H(t) R_{m}\left(U_{m-1}\right) \sin (t) d t\right)+c_{1} \sin (t)+c_{2} \cos (t), \\
U_{m}(0)= & 0, \quad U_{m}^{\prime}=0 .
\end{aligned}
$$

Equation (37) is the $m$ th order deformation equation for $x(t)$, where

$$
R_{m}\left(U_{m-1}\right)=\frac{d^{2} U_{m-1}(t)}{d t^{2}}+c^{2} U_{m-1}(t)+\varepsilon\left[\sum_{j=0}^{m-1} U_{m-1-j}(t) \sum_{z=0}^{j} U_{j-z}(t) U_{z}(t)\right],
$$

and

$$
\chi_{m}=\left\{\begin{array}{ll}
0, & m \leq 1 \\
1, & m>1
\end{array} .\right.
$$

As a result of this selection, the first and second terms of the solution's series are as follows:

$$
\begin{aligned}
& U_{0}(t)=A \cos (t), \\
& U_{1}(t)=\frac{1}{8} \sin (t) h A\left(-4 t+4 \alpha t+3 \lambda^{2} c^{2} t+\sin (t) \cos (t) \lambda^{2} \varepsilon\right) .
\end{aligned}
$$

The solution series $U(t)$ is developed up to 10th order of approximation.

To show the remarkable accuracy of the obtained result, a comparison is made in table 1 and figure 1 . It is shown that the accuracy of max-min and energy balance methods by one term is higher than HAM by two terms in series solution however, by increasing the order of HAM the accuracy increases. From the view point of engineering, computational cost and post processing, it is clear that working with a single term is easier than a long series with infinite terms obtained by HAM.

\subsection{Example 2}

It is considered that the simple mathematical pendulum which can be written in the following form when $u$ designates the deviation angle from the vertical equilibrium position, $\Omega^{2}=g / l$, where $g$ is the gravitational acceleration, $l$ the length of the pendulum (He 1999b). With initial conditions of:

$$
u^{\prime \prime}+\Omega^{2} \sin u=0, \quad u(0)=A, \quad u^{\prime}(0)=0 .
$$

The approximation of $\sin (u)$ is used:

$$
\sin u \approx u-\frac{u^{3}}{3 !}+\frac{u^{5}}{5 !}-\frac{u^{7}}{7 !}+\frac{u^{9}}{9 !} \cdots \rightarrow \sin u \approx u-\frac{u^{3}}{6}+\frac{u^{5}}{144}
$$


Consequently, as previous it is obtained:

$$
u(t)=A \cos \omega t, \quad u^{\prime \prime}(t)=-\omega^{2} u(t)
$$

by rewriting (45) in the form of:

$$
\begin{aligned}
-\omega^{2} u+\Omega^{2}\left(u-\frac{u^{3}}{6}+\frac{u^{5}}{144}\right)=0 & \Rightarrow \omega^{2}=\Omega^{2}\left(1-\frac{u^{2}}{6}+\frac{u^{4}}{144}\right)=\Omega^{2}\left(1-\frac{u^{2}}{12}\right)^{2} \\
& \Rightarrow \omega=\Omega\left(1-\frac{u^{2}}{12}\right) \Rightarrow 0<\omega<\Omega .
\end{aligned}
$$

According to the He Chengtian's interpolation:

$$
\frac{0}{1}<\omega<\frac{\Omega}{1} \text {. }
$$

So, by using the max-min to obtain the frequency, we have:

$$
\omega=\frac{m(0)+n(\Omega)}{m+n}=\frac{n \Omega}{m+n}=k \Omega
$$

where $m$ and $n$ are weighting factors, $k=n /(m+n)$.

So the frequency can be approximated as:

$$
\omega=k \Omega \text {. }
$$

And the approximate solution would be:

$$
u(t)=A \cos (k \Omega t),
$$

by rewriting (45) as in the previous example, it is obtained:

$$
u^{\prime \prime}+k^{2} \Omega^{2} u=\Omega^{2}\left(1-k^{2}\right) u-\Omega^{2} \frac{u^{3}}{6}+\Omega^{2} \frac{u^{5}}{144} .
$$

So if (50) is the exact solution, by vanishing the right hand side of (51), we have:

$$
B=\int_{0}^{T / 4}\left(\Omega^{2}\left(1-k^{2}\right) u-\Omega^{2} \frac{u^{3}}{6}+\Omega^{2} \frac{u^{5}}{144}\right) \cos \omega t d t=0
$$

by substituting (52) into (51), where $T=2 \pi / \omega$.

The sub-term of $B$ which equals to $B_{1}+B_{2}+B_{3}$, is obtained for simplification of the solution to readers, $B$ is divided as a Singular term located in the integral to some terms, which are then solved one by one in the manuscript so as to reduce the initial term to:

$$
\begin{aligned}
& \left\{\begin{array} { l } 
{ B _ { 1 } = \int _ { 0 } ^ { \pi / 2 } ( \Omega ^ { 2 } ( 1 - k ^ { 2 } ) A \operatorname { c o s } ^ { 2 } \omega t d t ) } \\
{ B _ { 2 } = \int _ { 0 } ^ { \pi / 2 } ( - \Omega ^ { 2 } \frac { A ^ { 3 } } { 6 } \operatorname { c o s } ^ { 4 } \omega t d t ) } \\
{ B _ { 3 } = \int _ { 0 } ^ { \pi / 2 } ( \Omega ^ { 2 } \frac { A ^ { 5 } } { 1 4 4 } \operatorname { c o s } ^ { 6 } \omega t d t ) }
\end{array} \Rightarrow \left\{\begin{array}{l}
B_{1}=\left(-A k^{2} \Omega^{2}+\Omega^{2} A\right) \frac{\pi}{4} \\
B_{2}=-\Omega^{2} \frac{\pi A^{3}}{32} \\
B_{3}=\Omega^{2} \frac{5 \pi A^{5}}{144 \times 32}
\end{array}\right.\right. \\
& B=A \Omega^{2} \frac{\pi}{4}\left(1-k^{2}-\frac{A^{2}}{8}+\frac{5 A^{4}}{144 \times 8}\right)=0 .
\end{aligned}
$$


So

$$
k=\sqrt{1-\frac{A^{2}}{8}+\frac{5 A^{4}}{1152}} .
$$

So the frequency is obtained, as follows.

$$
\omega=\sqrt{k^{2} \Omega^{2}}=\left[\left(1-\frac{A^{2}}{8}+\frac{5 A^{4}}{1152}\right)^{2} \times \Omega^{2}\right]^{1 / 2} .
$$

Moreover, the approximate period is:

$$
T=\frac{2 \pi}{\sqrt{\Omega^{2}\left(1-\frac{A^{2}}{8}+\frac{5 A^{4}}{1152}\right)}} .
$$

The result obtained from this example using max-min are compared with EBM results in table 2 and figure 3 and the phase plane has been shown in figure 4. Obviously, the comparison shows the accuracy of the obtained analytical solution (Babazadeh et al 2008).

\subsection{Example 3}

Consider the following nonlinear oscillator (Arnold 2006):

$$
u^{\prime \prime}+u+\varepsilon u^{5}=0 \quad u(0)=A, u^{\prime}(0)=0 .
$$

The trial-function is chosen as:

$$
u=A \cos \omega t,
$$

by rewriting (57) in the following form:

$$
u^{\prime \prime}+\left(1+\varepsilon u^{4}\right) u=0 .
$$

Table 2. Comparison between different methods to obtain the frequency $(\Omega=1)$.

\begin{tabular}{lccccc}
\hline A & $\begin{array}{c}\text { Energy balance } \\
\text { frequency }\end{array}$ & $\begin{array}{c}\text { Max-min } \\
\text { frequency }\end{array}$ & $\begin{array}{c}\text { HAM with } \\
1 \text { order }\end{array}$ & $\begin{array}{c}\text { HAM with } \\
10 \text { orders }\end{array}$ & $\begin{array}{c}\text { Numerical } \\
\text { solution }\end{array}$ \\
\hline 0.01 & 0.99999 & 0.99999 & 0.99999 & 0.99999 & 0.99999 \\
$0 \cdot 1$ & 0.99937 & 0.99937 & 0.99941 & 0.99937 & 0.99937 \\
$0 \cdot 2$ & 0.99750 & 0.99750 & 0.99739 & 0.99741 & 0.99742 \\
$0 \cdot 3$ & 0.99437 & 0.99436 & 0.99402 & 0.99429 & 0.99430 \\
0.4 & 0.98998 & 0.98996 & 0.98979 & 0.98984 & 0.98983 \\
$0 \cdot 5$ & 0.98433 & 0.98428 & 0.98400 & 0.98429 & 0.98422 \\
0.6 & 0.97740 & 0.97730 & 0.97691 & 0.97731 & 0.97736 \\
$0 \cdot 7$ & 0.96919 & 0.96900 & 0.96862 & 0.96902 & 0.96902 \\
0.8 & 0.95969 & 0.95935 & 0.95902 & 0.95950 & 0.95942 \\
0.9 & 0.94886 & 0.94832 & 0.94812 & 0.94859 & 0.94851 \\
1 & 0.93671 & 0.93588 & 0.93510 & 0.93612 & 0.93602 \\
1.5 & 0.85502 & 0.85038 & 0.85419 & 0.85418 & 0.85498 \\
\hline
\end{tabular}




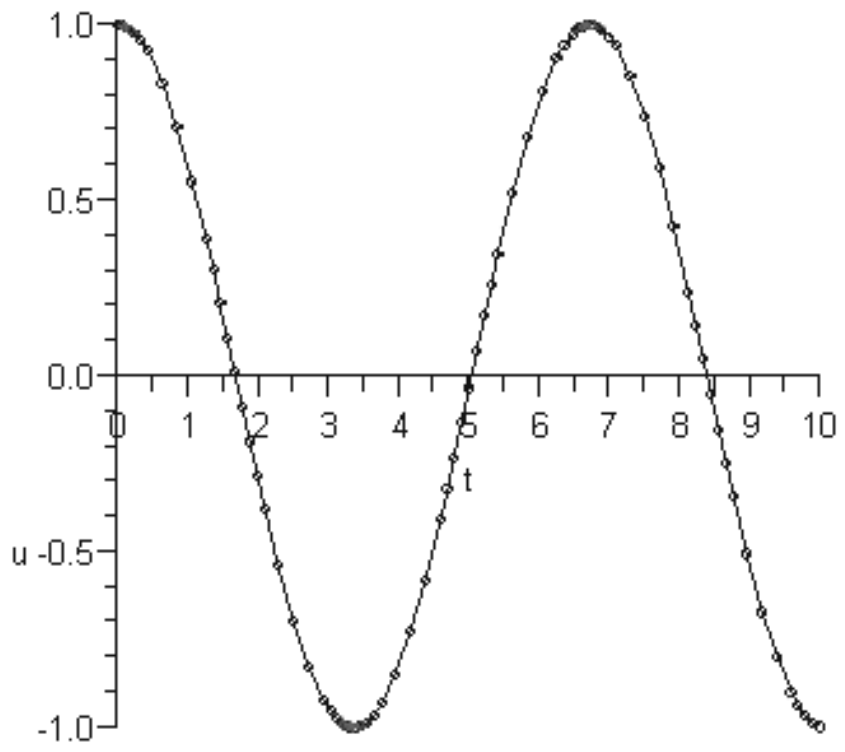

So we have:

$$
\begin{aligned}
& -\omega^{2} u+\left(1+\varepsilon u^{4}\right) u=0 \\
& \omega=\sqrt{1+\varepsilon u^{4}} \\
& \left\{\begin{array}{l}
\omega_{M}=\sqrt{1+\varepsilon A^{4}} \\
\omega_{m}=1
\end{array}\right.
\end{aligned}
$$

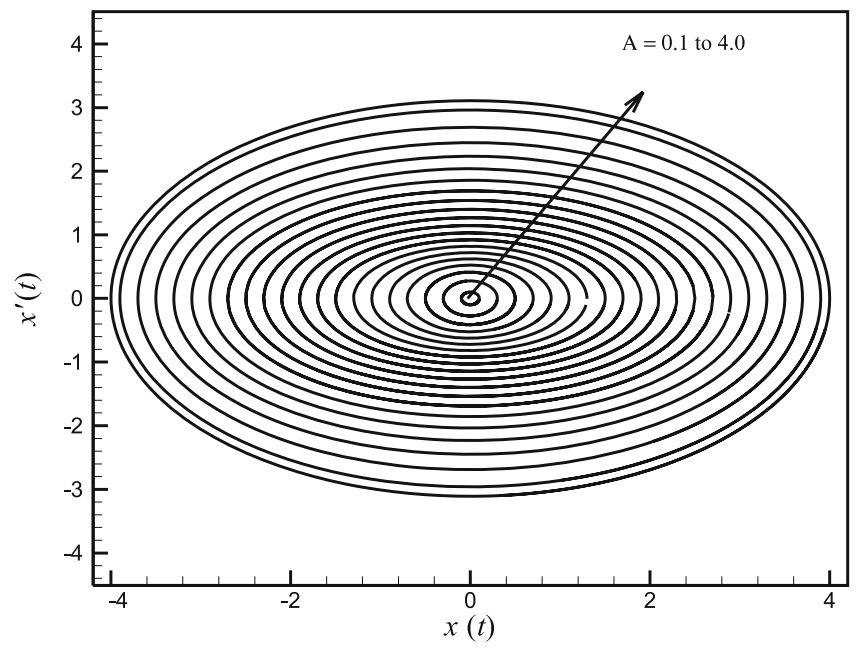

Figure 3. Comparison of the energy balance solution with the max-min solution; pointed line; energy balance and solid line; the max-min solution $(A=1)$.

Figure 4. Phase plane maps showing the system stability, influence of $A$ in the stability. 
According to the He Chengtian's interpolation, it is reduced to:

$$
\begin{aligned}
& \frac{1}{1}<\omega^{2}<\frac{1+\varepsilon A^{4}}{1} \\
& \omega^{2}=\frac{m(1)+n\left(1+\varepsilon A^{4}\right)}{m+n}=\left(1+k \varepsilon A^{4}\right),
\end{aligned}
$$

where $m$ and $n$ are weighting factors, $k=n /(m+n)$. So the frequency is approximated as:

$$
\omega=\sqrt{1+k \varepsilon A^{4}}
$$

Therefore, can be estimated as:

$$
u(t)=A \cos \left[\left(1+k \varepsilon A^{4}\right)^{1 / 2} t\right] .
$$

By substituting the solution into (57), we have:

$$
u^{\prime \prime}+\left(1+k \varepsilon A^{4}\right) u=k \varepsilon A^{4} u-\varepsilon u^{5} .
$$

If we assume (65) is the exact solution, the right side of (66) can be eliminated, so it can be written:

$$
B=\int_{0}^{T / 4}\left(k \varepsilon A^{4} u-\varepsilon u\right) \cos \omega t d t=0,
$$

by substituting (19) into (21), where $T=2 \pi / \omega$ according to previous examples, it is obtained:

$$
k=5 / 8 \text {. }
$$

Finally the frequency is:

$$
\omega=\sqrt{1+\frac{5}{8} \varepsilon A^{4}}
$$

Table 3. Comparison between different methods to obtain the frequency.

\begin{tabular}{lccccc}
\hline $\mathrm{A}$ & $\begin{array}{c}\text { Energy balance } \\
\text { frequency }\end{array}$ & $\begin{array}{c}\text { Max-min } \\
\text { frequency }\end{array}$ & $\begin{array}{c}\text { HAM with } \\
1 \text { order }\end{array}$ & $\begin{array}{c}\text { HAM with } \\
10 \text { orders }\end{array}$ & $\begin{array}{c}\text { Numerical } \\
\text { solution }\end{array}$ \\
\hline $0 \cdot 01$ & 1.0000000 & 1.0000000 & 1.0000000 & 1.0000000 & 1.0000000 \\
$0 \cdot 1$ & 1.0000003 & 1.0000003 & 1.0000002 & 1.0000010 & 1.0000010 \\
$0 \cdot 2$ & 1.0000047 & 1.0000050 & 1.0000031 & 1.0000058 & 1.0000058 \\
$0 \cdot 3$ & 1.0000236 & 1.0000253 & 1.0000298 & 1.0000235 & 1.0000236 \\
$0 \cdot 4$ & 1.0000747 & 1.0000800 & 1.0001290 & 1.0000660 & 1.0000662 \\
0.5 & 1.0001823 & 1.0001953 & 1.0001185 & 1.0001821 & 1.0001823 \\
$0 \cdot 6$ & 1.0003779 & 1.0004049 & 1.0005892 & 1.0002917 & 1.0002935 \\
$0 \cdot 7$ & 1.0007000 & 1.0007500 & 1.0006690 & 1.0007021 & 1.0007015 \\
$0 \cdot 8$ & 1.0011940 & 1.0012792 & 1.0015382 & 1.0010918 & 1.0010925 \\
0.9 & 1.0019118 & 1.0020482 & 1.0018920 & 1.0011902 & 1.0011935 \\
1 & 1.0029124 & 1.0031201 & 1.0035400 & 1.0029184 & 1.0029124 \\
1.5 & 1.0146582 & 1.0156971 & 1.0295400 & 1.0110893 & 1.0110945 \\
\hline
\end{tabular}




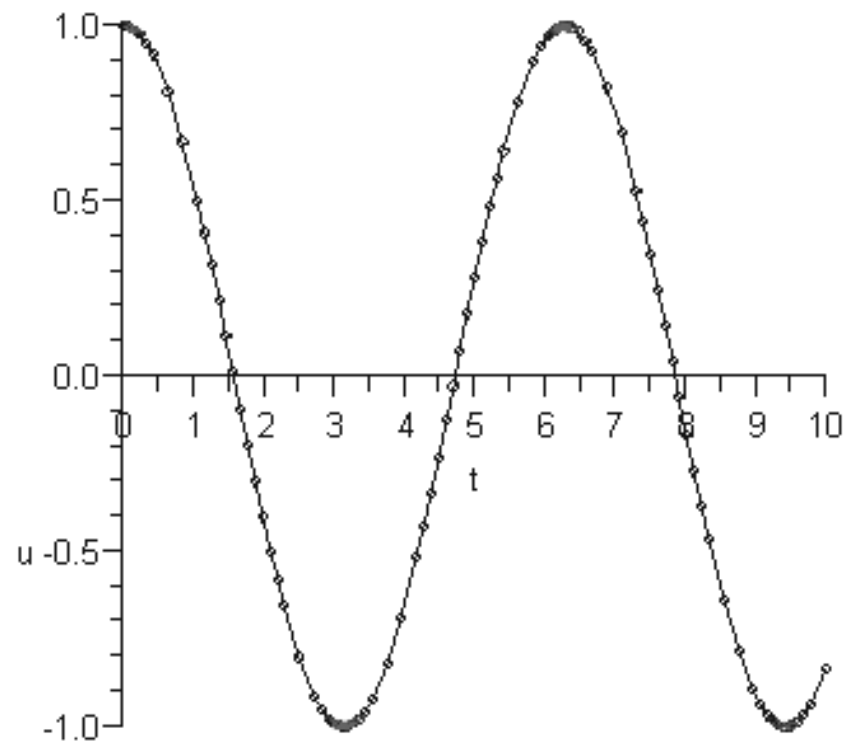

Figure 5. Comparison of the energy balance solution with the max-min solution; pointed line; energy balance and solid line; the max-min solution $(A=0.6)$.

and the approximate period is:

$$
T=2 \pi / \sqrt{1+\frac{5}{8} \varepsilon A^{4}} .
$$

The comparison of the max-min solution with EBM solution (Babazaeh et al 2008) and HAM are given in table 3 and figure 5 and the phase plane is shown in figure 6 . The figure 5 shows good agreement between the results.

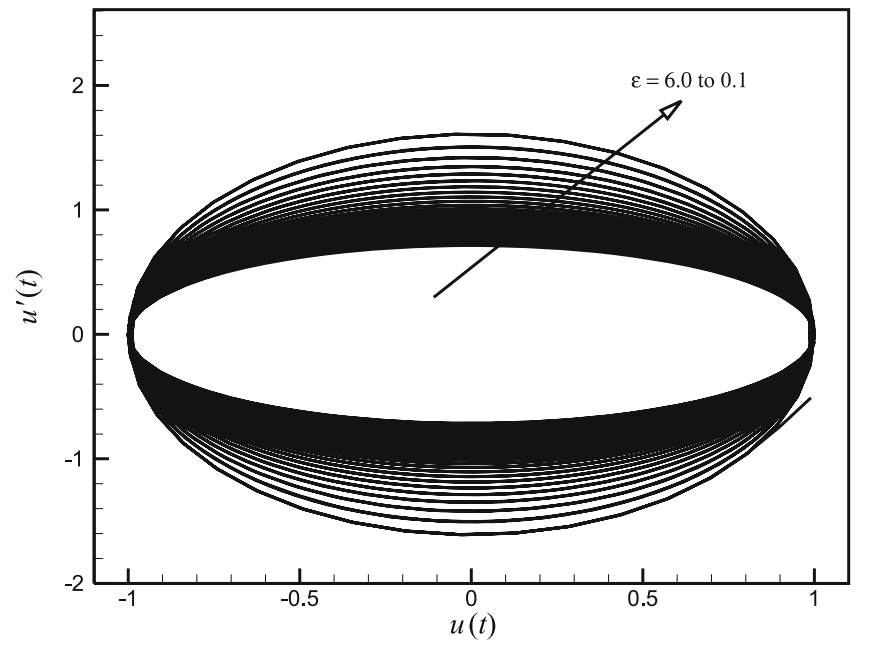

Figure 6. Phase plane maps showing the system stability, influence of $\varepsilon$ in the stability. 


\section{Conclusion}

He's max-min method was successfully applied to some strong nonlinear equations such as pendulum and duffing problems. In addition, accuracy of the method was investigated by a comparison which was made between HAM, energy balance and numerical solution. It was shown that the accuracy of the method with only one term calculated is higher than HAM by two orders in series solution. The method is useful to obtain analytical solution for all oscillators and vibration problems, such as in the fields of civil structures, fluid mechanics, electromagnetics and waves, etc. Oscillator problems are very frequently encountered in all of the mentioned major fields of science and engineering. The max-min method provides an easy and direct procedure for determining approximations to the periodic solutions. The max-min method is a well-established method for analysing nonlinear systems which can be easily extended to any nonlinear equation. The accuracy and efficiency of the method was demonstrated by presenting some examples.

\section{References}

Arnold M D 2006 An efficient solution for scattering by a perfectly conducting strip grating. J. Electromagnetic Waves and Applications 20: 891-900

Babazadeh H, Ganji D D, Akbarzade M 2008 He's energy Balance method to evaluate the effect of amplitude on the natural frequency in nonlinear vibration systems. Progress in Electromagnetics Res. 4: 143-154

Barari A, Omidvar M, Ghotbi Abdoul R, Ganji D D 2008 Application of homotopy perturbation method and variational iteration method to nonlinear oscillator differential equations. Acta Applicandae Mathematicae. 104: 161-171

Bender C M, Pinsky K S, Simmons L M 1989 A new perturbative approach to nonlinear problems. J. Mathematical Physics. 30: 1447-1455

Catal S 2008 Solution of free vibration equations of beam on elastic soil by using differential transform method. Applied Mathematical Modelling 32: 1744-1757

Dehghan M, Shakeri F 2008 The use of the decomposition procedure of Adomian for solving a delay differential equation arising in electrodynamics. Physica Scripta 78: 1-11

Fooladi M, Abaspour S R, Kimiaeifar A, Rahimpour M 2009 On the analytical solution of nonlinear normal mode for continuous systems by means of HAM. World Applied Sciences J. 6: 297-302

Ganji S S, Ganji D D, Babazadeh H, Sadoughi N 2009 Application of amplitude-frequency formulation to nonlinear oscillation system of the motion of a rigid rod rocking back. Mathematical Methods in the Applied Sciences 33: 157-166

Hamdan M N, Shabaneh N H 1997 On the large amplitude free vibrations of a restrained uniform beam carrying an intermediate lumped mass. J. Sound and Vibration 199: 711-736

Hashemi Kachapi SHA, Barari A, Tolou N, Ganji D D 2009 Solution of strongly nonlinear oscillation systems using variational approach. J. Applied Functional Analysis 4: 528-535

He J H, Wu G C, Austin F 2010 The variational iteration method which should be followed. Nonlinear Sci. Lett. A 1: 1-30

He J H 2001 Modified Lindstedt-Poincare methods for some strongly nonlinear oscillations. Part III: Double series expansion. Inter. J. Nonlinear Sciences and Numerical Simulation 2: 317-320

He J H 2002a A note on delta-perturbation expansion method. Applied Mathematics and Mechanics 23: 634-638

He J H 2002b Modified Lindstedt-Poincare methods for some strongly nonlinear oscillations. Part I: expansion of a constant. Inter. J. Non-linear Mechanic 37: 309-314

He J H 2004 The homotopy perturbation method for nonlinear oscillators with discontinuities. Applied Mathematics and Computation 151: 287-292 
He J H 1999a Some new approaches to Duffing equation with strongly and high order nonlinearity (II) parameterized perturbation technique. Communications in Nonlinear Science and Numerical Simulation 4: 81-83

He J H 2006a Determination of limit cycles for strongly nonlinear oscillators. Phys. Rev. Lett. 90: $174-176$

He J H 2007 Variational approach for nonlinear oscillators. Chaos, Solitons and Fractals. 34: $1430-1439$

He J H 2009 Application of He Chengtian's interpolation to Bethe equation. Computers \& Mathematics with Applications 58: 2427-2430

He J H 2006b Non-pertubative methods for strongly nonlinear problems. dissertation. de-Verlag im Internet $\mathrm{GmbH}$

He J H 1999b Variational iteration method: A kind of nonlinear analytical technique: Some examples. Inter. J. Nonlinear Mechanics 34: 699-708

He J H 2008 Max-min approach to nonlinear oscillators. Inter. J. Nonlinear Sciences and Numerical Simulation 9: $207-210$

Herisanu N, Marinca V 2010 A modified variational iteration method for strongly nonlinear oscillators. Nonlinear Sci. Lett. A 1: 183-192

Ji Z 1999 Mathematics in Northern-Southern, Sui and Tang Dynasties (Shijiazhuang: Hubei Science and Technology Publishing House) (in Chinese)

Kimiaeifar A, Saidi A R, Sohouli A R, Ganji D D 2010 Analysis of modified Van der Pol's oscillator using He's parameter expanding method. Current Applied Physics 10: 279-283

Kimiaeifar A 2010 An analytical approach to investigate the response and stability of Van der PolMathieu-Duffing oscillators under different excitation functions. J. Mathematical Methods in the Applied Sciences DOI: 10.1002/mma.1269

Kimiaeifar A, Saidi A R, Bagheri G H, Rahimpour M, Domairry D G 2009a Analytical solution for Van der Pol-Duffing oscillators. Chaos, Solitons and Fractals 42: 2660-2666

Kimiaeifar A, Bagheri G H, Rahimpour M, Mehrabian M A 2009b Analytical solution of twodimensional stagnation flow towards a shrinking sheet by means of Homotopy analysis method. J. Process Mechanical Eng. 223: 133-143

Lai S K, Lim C W, Wu B S, Wang C, Zeng Q C, He X F 2009 Newton-harmonic balancing approach for accurate solutions to nonlinear Cubic-Quintic Duffing oscillators. Applied Mathematical Modelling 33: 852-866

Mohammadi M H, Mohammadi A, Kimiaeifar A, Tabaei H 2009 Application of HPEM to find an analytical solution for single degree of freedom problems in nonlinear vibration. Inter. J. Applied Mathematics and Mechanics 5: 80-87

Momeni M, Jamshidi N, Barari A, Ganji D D 2010 Application of He's energy Balance method to Duffing harmonic oscillators. Inter. J. Computer Mathematics DOI: 10.1080/00207160903337239

Okuizumi N, Kimura K 2004 Multiple time scale analysis of hysteretic systems subjected to harmonic excitation. J. Sound and Vibration 272: 675-701

Özis T, Yildirim A 2007a A comparative study of He's homotopy perturbation method for determining frequency-amplitude relation of a nonlinear oscillator with discontinuities. Inter. J. Nonlinear Sciences and Numerical Simulation 8: 243-248

Özis T, Yildirim A 2007b Determination of periodic solution for a $u^{1 / 3}$ force by He's modified lindstedt-poincaré method. J. Sound and Vibration 301: 415-419

Qian B C 1992 History of Chinese mathematics (Beijing: Science Publisher) (in Chinese)

Shen Y Y, Mo L F 2009 The max-min approach to a relativistic equation. Computers \& Mathematics with Applications. 58: 2131-2133

Tolou N, Hashemi Kachapi S H A, Barari A, Ganji D D 2009 Analytical investigation of strongly nonlinear normal mode using homotopy perturbation method and He's variational method. J. Applied Functional Analysis 4: 682-690

Xu L 2008 Variational approach to solitons of nonlinear dispersive $K(m, n)$ equations. Chaos, Solitons \& Fractals 37: 137-143 
Zeng D Q, Lee Y Y 2009 Analysis of strongly nonlinear oscillator using the max-min approach. Inter. J. Nonlinear Sciences and Numerical Simulation 10: 1361-1368

Zeng D Q 2009 Nonlinear oscillator with discontinuity by the max-min approach. Chaos, Solitons \& Fractals 42: 2885-2889 